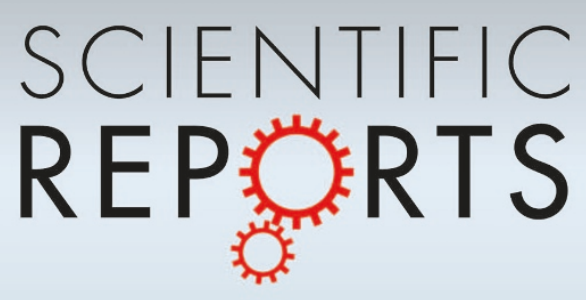

OPEN

SUBJECT AREAS:

QUANTUM PHYSICS

INFORMATION THEORY AND

COMPUTATION

MATHEMATICS

THEORETICAL PHYSICS

Received

29 May 2012

Accepted

21 June 2012

Published

13 July 2012

Correspondence and requests for materials should be addressed to T.M. (morimae@gmail. com) or

K.F. (keisukejayorz@ gmail.com)

\section{Not all physical errors can be linear CPTP maps in a correlation space}

\author{
Tomoyuki Morimae ${ }^{1,2,3}$ \& Keisuke Fujii ${ }^{4}$
}

${ }^{1}$ Controlled Quantum Dynamics Theory Group, Imperial College London, London SW7 2AZ, United Kingdom, ${ }^{2}$ LAMA, Université Paris-Est Marne-la-Vallée, 77454 Marne-la-Vallée Cedex 2, France, ${ }^{3}$ IRCS, Tokyo Institute of Technology, 2-12-1 Ookayama, Meguro-ku, Tokyo 152-8550, Japan, ${ }^{4}$ Graduate School of Engineering Science, Osaka University, Toyonaka, Osaka 560-853 1, Japan.

In the framework of quantum computational tensor network, which is a general framework of measurement-based quantum computation, the resource many-body state is represented in a tensor-network form (or a matrix-product form), and universal quantum computation is performed in a virtual linear space, which is called a correlation space, where tensors live. Since any unitary operation, state preparation, and the projection measurement in the computational basis can be simulated in a correlation space, it is natural to expect that fault-tolerant quantum circuits can also be simulated in a correlation space. However, we point out that not all physical errors on physical qudits appear as linear completely-positive trace-preserving errors in a correlation space. Since the theories of fault-tolerant quantum circuits known so far assume such noises, this means that the simulation of fault-tolerant quantum circuits in a correlation space is not so straightforward for general resource states.

Q uantum many-body states, which have long been central research objects in condensed matter physics, statistical physics, and quantum chemistry, are now attracting the renewed interest in quantum information science as fundamental resources for quantum information processing. One of the most celebrated examples is one-way quantum computation ${ }^{1-3}$. Once the highly-entangled many-body state which is called the cluster state is prepared, universal quantum computation is possible with adaptive local measurements on each qubit. Recently, the concept of quantum computational tensor network $(\mathrm{QCTN})^{4-6}$, which is the general framework of measurement-based quantum computation on quantum many-body states, was proposed. This novel framework has enabled us to understand how general measurement-based quantum computation is performed on many other resource states beyond the cluster state. The most innovative feature of QCTN is that the resource state is represented in a tensor-network form (or a matrix-product form $)^{7-9}$, and universal quantum computation is performed in the virtual linear space where tensors live. For example, let us consider the one-dimensional openboundary chain of $N$ qudits in the matrix-product form

$$
|\Psi\rangle \equiv \sum_{k_{1}=0}^{d-1} \ldots \sum_{k_{N}=0}^{d-1}\left\langle L\left|A\left[k_{N}\right] \ldots A\left[k_{1}\right]\right| R\right\rangle\left|k_{N}, \ldots, k_{1}\right\rangle,
$$

where $\{|0\rangle, \ldots,|d-1\rangle\}$ is a certain basis in the $d$-dimensional Hilbert space $(2 \leq d<\infty),|L\rangle$ and $|R\rangle$ are $D$ dimensional complex vectors, and $\{A[0], \ldots, A[d-1]\}$ are $D \times D$ complex matrices. Let us also define the projection measurement $\mathcal{M}_{\theta, \phi}$ on a single physical qudit by

$$
\mathcal{M}_{\theta, \phi} \equiv\left\{\left|\alpha_{\theta, \phi}\right\rangle,\left|\beta_{\theta, \phi}\right\rangle,|2\rangle, \ldots,|d-1\rangle\right\},
$$

where

$$
\begin{aligned}
\left|\alpha_{\theta, \phi}\right\rangle & \equiv \cos \frac{\theta}{2}|0\rangle+e^{i \phi} \sin \frac{\theta}{2}|1\rangle, \\
\left|\beta_{\theta, \phi}\right\rangle & \equiv \sin \frac{\theta}{2}|0\rangle-e^{i \phi} \cos \frac{\theta}{2}|1\rangle,
\end{aligned}
$$

$0<\theta<\pi$, and $0 \leq \phi<2 \pi$. If we do the measurement $\mathcal{M}_{\theta, \phi}$ on the first physical qudit of Eq. (1) and if the first physical qudit is projected onto, for example, $\left|\alpha_{\theta, \phi}\right\rangle$ as a result of this measurement, the state Eq. (1) becomes 


$$
\sum_{k_{2}, \ldots, k_{N}}\left\langle L\left|A\left[k_{N}\right] \ldots A\left[k_{2}\right] \frac{A\left[\alpha_{\theta, \phi}\right]}{\left\|A\left[\alpha_{\theta, \phi}\right]\right\|}\right| R\right\rangle\left|k_{N}, \ldots, k_{2}\right\rangle \otimes\left|\alpha_{\theta, \phi}\right\rangle,
$$

where

$$
A\left[\alpha_{\theta, \phi}\right] \equiv \cos \frac{\theta}{2} A[0]+e^{-i \phi} \sin \frac{\theta}{2} A[1] .
$$

Now we can imagine a virtual linear space where $A$ 's, $|R\rangle$, and $|L\rangle$ live. This virtual linear space is called the correlation space ${ }^{4-6,10-12}$. Then, the above equation can be interpreted that the "gate operation" $|R\rangle \rightarrow \frac{A\left[\alpha_{\theta, \phi}\right]}{\left\|A\left[\alpha_{\theta, \phi}\right]\right\|}|R\rangle$ is implemented on the "quantum state" $|R\rangle$ in the correlation space. In particular, if $A[0], A[1], \theta$, and $\phi$ are appro-

priately chosen in such a way that $A\left[\alpha_{\theta, \phi}\right]$ is proportional to a unitary, we can "simulate" the unitary evolution $\frac{A\left[\alpha_{\theta, \phi}\right]}{\left\|A\left[\alpha_{\theta, \phi}\right]\right\|}|R\rangle$ of the vector $|R\rangle$ in the correlation space. The core of QCTN is this "virtual quantum computation" in the correlation space. If the correlation space has a sufficient structure and if $A$ 's, $|L\rangle$, and $|R\rangle$ are appropriately chosen, we can "simulate" universal quantum circuit in the correlation space $e^{4-6,10-12}$.

For the realization of a scalable quantum computer, a theory of fault-tolerant (FT) quantum computation ${ }^{13-17}$ is necessary. In fact, several researches have been performed on FT quantum computation in the one-way model ${ }^{3,18-22}$. However, there has been no result about a theory of FT quantum computation on general QCTN. (See the recent progress to this direction ${ }^{23-26}$.) In particular, there is severe lack of knowledge about FT quantum computation on resource states with $d \geq 3$. It is necessary to consider resource states with $d \geq 3$ if we want to enjoy the cooling preparation of a resource state and the energy-gap protection of measurement-based quantum computation with a physically natural Hamiltonian, since no genuinely entangled qubit state can be the unique ground state of a two-body frustration-free Hamiltonian ${ }^{27}$.

One straightforward way of implementing FT quantum computation on QCTN is to encode physical qudits with a quantum error correcting code:

$$
\sum_{k_{1}=0}^{d-1} \ldots \sum_{k_{N}=0}^{d-1}\left\langle L\left|A\left[k_{N}\right] \ldots A\left[k_{1}\right]\right| R\right\rangle\left|\tilde{k}_{N}, \ldots, \tilde{k}_{1}\right\rangle,
$$

where $\left|\tilde{k}_{i}\right\rangle(i=1, \ldots, N)$ is the encoded version of $\left|k_{i}\right\rangle$ (such as $|\tilde{0}\rangle=|000\rangle$ and $|\tilde{1}\rangle=|111\rangle$, etc.) In fact, this strategy was taken in Refs. $^{21,22}$ for the one-way model $(d=2)$, and it was shown there that a FT construction of the encoded cluster state is possible. For $d \geq 3$, however, such a strategy is difficult, since theories of quantum error correcting codes and FT preparations of the encoded resource state are less developed for $d \geq 3$. Furthermore, if we encode physical qudits with a quantum error correcting code, the parent Hamiltonian should no longer be two-body interacting one.

The other way of implementing FT quantum computation on QCTN is to simulate FT quantum circuits in the correlation space. Since any unitary operation, state preparation, and the projective measurement in the computational basis can be simulated in a correlation space (for a precise discussion about the possibility of the measurement, see Ref. ${ }^{12}$ ), it is natural to expect that FT quantum circuits can also be simulated in a correlation space. An advantage of this strategy is that theories of FT quantum circuits for qubit systems are well developed ${ }^{13-17}$. In fact, this strategy was taken in Refs. ${ }^{18,19}$ for the one-way model $(d=2)$. They introduced a method (which we call "the ensemble method" since the ensemble of all measurement results are considered) of simulating quantum circuits in the correlation space of the cluster state, and showed that all physical errors on physical qubits can be linear completely-positive trace-preserving (CPTP) maps in the correlation space of the cluster state. (For details, see Sec. I of the supplementary material.) This means that FT quantum circuits can be simulated in the correlation space of the cluster state.

In this paper, however, we point out that it is not so straightforward to simulate FT quantum circuits in the correlation space of a general resource state. In order to see it, we consider two standard methods of simulating quantum circuits in the correlation space. First, we directly apply the ensemble method of Refs. ${ }^{18,19}$ to the one-dimensional Affleck-Kennedy-Lieb-Tasaki (AKLT) state ${ }^{28,29}$, as an example, and see that not all physical errors on physical qutrits can be linear CPTP maps in the correlation space of the AKLT state. Since all theories of FT quantum circuits known so far assume such noises ${ }^{13-17}$, this means that it is not so straightforward to apply these FT theories to quantum circuits simulated in the correlation space of general resource states. (Note that quantum error correction is not equivalent to fault-tolerant quantum computation: non-CPTP errors can be corrected by quantum error-correcting codes, but that does not necessarily mean the possibility of fault-tolerant quantum computation.) We give some intuitive explanations of reasons why the cluster state is so special and why not all resource states work as the cluster state. Second, we consider another natural method of simulating quantum circuits in the correlation space, which we call "the trajectory method" (since only a specific history of measurement results, i.e., a specific trajectory, is considered). This method is another standard way of simulating quantum circuits in the correlation space when the system is assumed to be error-free $e^{4-6,10,28}$. We show a general theorem which says that if $d \geq 3$ not all physical errors on physical qudits can be linear CPTP maps in the correlation space if we consider the trajectory method. This means that the trajectory method does neither work in general.

In short, we show in this paper that it is not so straightforward to simulate FT quantum circuits in the correlation space of a general resource state. Since all errors behave nicely in the correlation space of the cluster state ${ }^{18,19}$, less attention has been paid to the difference between a real physical space and a correlation space of a general resource state. Our results here suggest that these two spaces can be different, and because of the difference, simulations of FT quantum circuits can be difficult in a general correlation space. To our knowledge, there is so far no other methods than those two methods, i.e., the ensemble method and the trajectory method. However, of course there is a possibility that a new method will be developed in a future. Therefore, what we show here is not the impossibility of making QCTN fault-tolerant. Such a new method might be able to make all QCTN fault-tolerant. We hope that our results here will help to tackle such a challenging subject of a future study.

\section{Results}

Assumption. Throughout this paper, we make the following assumptions: Since the MPS $|\Psi(L, R)\rangle_{1}^{N}$, Eq. (1), is a resource state for measurement-based quantum computation, we can assume without loss of generality that $A\left[\alpha_{\theta, \phi}\right], A\left[\beta_{\theta, \phi}\right], A[2], A[3], \ldots, A[d$ $-1]$ are unitary up to constants:

$$
\begin{aligned}
A\left[\alpha_{\theta, \phi}\right] & =c_{\alpha} U_{\alpha}, \\
A\left[\beta_{\theta, \phi}\right] & =c_{\beta} U_{\beta}, \\
A[2] & =c_{2} U_{2}, \\
A[3] & =c_{3} U_{3}, \\
\cdots & \\
A[d-1] & =c_{d-1} U_{d-1},
\end{aligned}
$$

where $c_{\alpha}, c_{\beta}, c_{2}, \ldots c_{d-1}$ are real positive numbers, $U_{\alpha}, U_{\beta}, U_{2}, \ldots$, $U_{d-1}$ are unitary operators, and 


$$
A\left[\beta_{\theta, \phi}\right] \equiv \sin \frac{\theta}{2} A[0]-e^{-i \phi} \cos \frac{\theta}{2} A[1]
$$

This means that any operation implemented in the correlation space by the measurement $\mathcal{M}_{\theta, \phi}$ on a single physical qudit of $|\Psi(L, R)\rangle_{1}^{N}$ is unitary. Note that this assumption is reasonable, since otherwise $|\Psi(L, R)\rangle_{1}^{N}$ does not seem to be useful as a resource for measurement-based quantum computation. In fact, all known resource states so far including the cluster state and the AKLT state, satisfy this assumption by appropriately rotating each local physical basis. Furthermore, we can take $c_{\alpha}, c_{\beta}, c_{2}, \ldots, c_{d-1}$ such that

$$
C \equiv c_{\alpha}^{2}+c_{\beta}^{2}+\sum_{k=2}^{d-1} c_{k}^{2}=1,
$$

since

$$
\begin{aligned}
& \frac{1}{\sqrt{f_{N}(|L\rangle,|R\rangle)}} \sum_{k_{1}, \ldots, k_{N}}\left\langle L\left|A\left[k_{N}\right] \ldots A\left[k_{1}\right]\right| R\right\rangle\left|k_{N}, \ldots, k_{1}\right\rangle \\
& =\frac{1}{\sqrt{f_{N}(|L\rangle,|R\rangle)}} \sqrt{C}^{N} \sum_{k_{1}, \ldots, k_{N}}\left\langle L\left|\frac{A\left[k_{N}\right]}{\sqrt{C}} \ldots \frac{A\left[k_{1}\right]}{\sqrt{C}}\right| R\right\rangle\left|k_{N}, \ldots, k_{1}\right\rangle
\end{aligned}
$$

and we can redefine $A\left[k_{i}\right] / \sqrt{C} \rightarrow A\left[k_{i}\right]$.

Simulation on the AKLT state with the ensemble method. Let us first consider the direct application of the ensemble method of Refs. ${ }^{18,19}$, which was used for the cluster state, to the AKLT state. As is shown in Refs. ${ }^{18,19}$, all physical errors can be linear CPTP maps in the correlation space of the cluster state. (This is also the case for the tricluster state ${ }^{30}$. See Sec. III and IV of the supplementary material.) However, here we show that if we directly apply the ensemble method to the AKLT state, not all physical errors can be linear CPTP maps in the correlation space of the AKLT state.

Roughly speaking, in the ensemble method, we consider the mixture of all measurement results (measurement histories) during the feed-forwarding stage. For the cluster state, it works well, since the desired gate operation can be implemented deterministically because of the feed-forwarding, and if we trace out states which registered each measurement results after the feed-forwarding, the entire state again becomes pure. (For details, see Ref. ${ }^{19}$ and the Supplementary Material.)
The one-dimensional AKLT state is the matrix-product state defined by $d=3, A[0]=X, A[1]=X Z$, and $A[2]=Z$. Let us assume that a CPTP error $\rho \rightarrow \sum_{j=1}^{w} F_{j} \rho F_{j}^{\dagger}$, where $\sum_{j=1}^{w} F_{j}^{\dagger} F_{j}=I$, occurs on a physical qutrit of the AKLT state. If we simulate quantum circuits in the correlation space of the AKLT state after this error with the ensemble method, the map

$$
|R\rangle\left\langle R\left|\rightarrow \mathcal{E}_{A K L T}(|R\rangle\langle R|) \equiv \sum_{j} \Xi_{j}\right| R\right\rangle\langle R| \Xi_{j}^{\dagger}
$$

is implemented in the correlation space, where $\Xi_{j}$ 's are certain operators which depend on $\left\{F_{j}\right\}$. (For details, see Sec. II of the supplementary material.) For example, let us consider the error with $w=1$ and $F_{1}=\frac{1}{\sqrt{2}}\left(\left|\alpha_{\theta, \phi}\right\rangle+\left|\beta_{\theta, \phi}\right\rangle\right)\left\langle 0\left|-\frac{1}{\sqrt{2}}\left(\left|\alpha_{\theta, \phi}\right\rangle-\left|\beta_{\theta, \phi}\right\rangle\right)\right.\right.$ $\langle 1|+| 2\rangle\langle 2|$. Then, we can show that

$$
\sum_{j} \Xi_{j}^{\dagger} \Xi_{j}=\delta I+\frac{2}{3}|1\rangle\langle 1|,
$$

where $\delta$ is a certain positive number. This means that the map $\mathcal{E}_{A K L T}$ is not linear CPTP.

Intuitive explanations. We have seen that the ensemble method of Refs. ${ }^{18,19}$ for the cluster state cannot be directly applied to other resource states, such as the one-dimensional AKLT state. Why the cluster state is so special? And why it does not work for other resource states? Although the complete answer to these questions is beyond the scope of the present paper, since the study of QCTN itself has not been fully developed (for example, no one knows the necessary and sufficient condition for tensor-network states to be universal resource states), let us try to give some intuitive explanations here.

Figure 1 illustrates the reason why all physical errors become CPTP maps in the correlation space of the one-dimensional cluster state. Let us first consider the ideal case (a) where there is no error. The actual protocol (a-1) is mathematically equivalent to the "inputoutput" picture (a-2) where the physical input state $|\psi\rangle$ is teleported into the left-edge of the short-length cluster chain (indicated in yellow) and finally the physical output state $\left|\psi^{\prime}\right\rangle$ is extracted from the right-edge of the short-length cluster chain (indicated in yellow). Since both $|\psi\rangle$ and $\left|\psi^{\prime}\right\rangle$ are physical states, what is going on in the correlation space which maps the input state $|\psi\rangle$ to the output state

(a) Ideal case

(1) actual protocol

\section{$|\psi\rangle$}

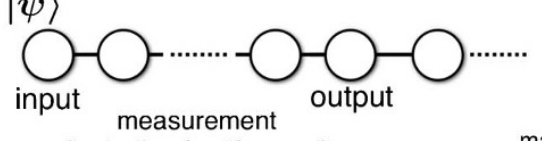

(including feedforward)

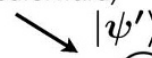

(2) "input-output" picture

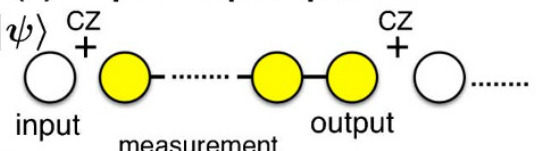

measurement

(including feedforward)

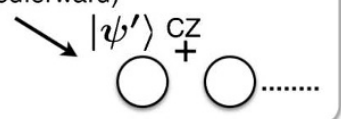

(b) Imperfect case (1) actual protocol

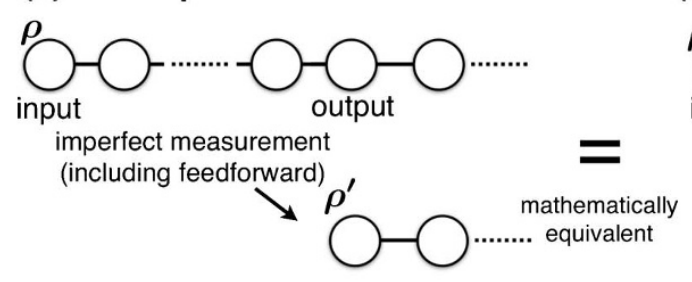

imperfection

(2) “input-output" picture

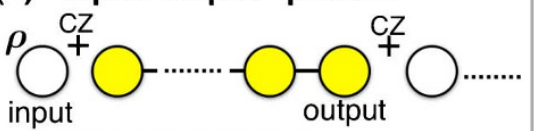

imperfect measurement (including feedforward)

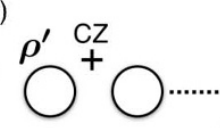

Figure 1 The "input-output" picture for the one-dimensional cluster state. 
$\left|\psi^{\prime}\right\rangle$ can be described by a linear CPTP operation. In other words, if we can describe measurement-based quantum computation with this "input-output" picture ${ }^{19}$, the map implemented in the correlation space is guaranteed to be a linear CPTP operation ${ }^{19}$.

For the cluster state, this "input-output" picture also holds even if there is an error ${ }^{19}$ : In the imperfect case, Fig. 1 (b), let us assume that the input state is degraded by an error and becomes a mixed state $\rho$. We also assume that measurements are imperfect. However, we can still consider a similar "input-output" picture (b-2) $)^{19}$, which corresponds to the actual protocol (b-1), and again the physical state $\rho$ is mapped into another physical state $\rho^{\prime}$, which means that what is going on in the correlation space which maps $\rho$ to $\rho^{\prime}$ can be described by a linear CPTP operation.

Note that two special properties of the cluster state enable such an "input-output" picture. First, the one-dimensional cluster state can be decomposed into small pieces of one-dimensional cluster states by applying nearest-neighbour two-body unitary operations (i.e., $\mathrm{CZ}$ gates). As we can see from Fig. 1 (a-2) and (b-2), this property is necessary for allowing the "input-output" picture. Second, the number of qubits that are measured in order to implement a specific gate does not depend on the measurement results. In other words, for the cluster state, a specific gate can be implemented up to Pauli byproducts at a fixed site irrespective of measurement results. Such a deterministic implementation of a gate at a fixed site is necessary for the deterministic (i.e., trace-preserving) "output" in the "input-output" picture, since in the ensemble method, the ensemble (mixture) of all measurement results are considered: If the site where the desired gate operation is completed depends on the measurement results, we cannot extract the same output state at a fixed site irrespective of measurement results as is shown in Fig. $1(a-2)$ and (b-2).

On the other hand, such an "input-output" picture seems to be impossible for the one-dimensional AKLT state, because of the following two reasons: First, as is shown in Fig. 2 left, no nearest-neighbour two-body unitary operation can decompose the one-dimensional AKLT chain into two chains due to the existence of the non-vanishing two-point correlation in the AKLT state. (If the one-dimensional AKLT chain can be decomposed into two chains by such a unitary, it contradicts to the well-known fact that the two-point correlation is non-vanishing in the AKLT state.) Second, we cannot deterministically implement a specific gate at a fixed site of the AKLT chain irrespective of measurement results ${ }^{4,5,28}$. In short, the "input-output" picture seems to be impossible for the AKLT state. If we can no longer use the "inputoutput" picture, it is not unreasonable that we have some anomalous maps in the correlation space since the correlation space is not a physical space but an abstract mathematical space.

Simulating quantum circuits with the trajectory method. As we have seen, it is not always possible for general resource states to implement a specific gate at a fixed site irrespective of measurement results. This fact prohibits a general resource state from allowing the "input-output" picture. One might think that if we abandon such a deterministic "output" at a fixed site for all measurement results, and if we just consider a specific history (trajectory) of measurement results, we might be able to avoid the emergence of non-CPTP errors, such as $\mathcal{E}_{A K L T}$. (Physically, this means that we project the system onto a pure state at every measurement step:

$$
|\Psi\rangle \rightarrow \frac{P|\Psi\rangle}{\| P|\Psi\rangle \|}
$$

with the corresponding projector $P$. Note that considering a specific trajectory of certain measurement results does not mean that if we obtain other trajectories, such events are discarded as done in, for example, linear optical quantum computation. Rather, it means that we describe measurement-based quantum computation with respect to each trajectory instead of the ensemble of all trajectories.) If the system is assumed to be error-free, this "trajectory method" is another standard way of simulating quantum circuits in the correlation space $e^{4-6,10,28}$. (Note that in this trajectory method, correct unitary operators can be implemented in the correlation space if there is no error, although what we are physically doing are projections, i.e., non-trace-preserving operations).

However, we here show that such a natural another way of simulating quantum circuits does neither work if $d \geq 3$. In other words, we can show the following theorem. (For a proof, see Methods.)

Theorem: If $d \geq 3$, there exists a single-qudit CPTP error $\mathcal{E}$ which has the following property: assume that $\mathcal{E}$ is applied on a single physical qudit of Eq. (1). If the measurement $\mathcal{M}_{\theta, \phi}$ is performed on that affected qudit, and if the entire system is projected onto a pure state as a result, a non-TP operation is implemented in the correlation space.

\section{Discussion}

In order to gain an intuitive understanding of the above theorem, let us finally consider the tensor-network state proposed in Ref. ${ }^{31}$ as an example. The state is defined by the following tensor network ${ }^{31}$ :

$$
\begin{gathered}
A_{\top}\left[+\frac{3}{2}\right]=|1\rangle\langle 0| \otimes\langle 1|, \\
A_{\top}\left[-\frac{3}{2}\right]=|0\rangle\langle 1| \otimes\langle 0|, \\
A_{\top}\left[+\frac{1}{2}\right]=-\frac{1}{\sqrt{3}}(Z \otimes\langle 1|+| 1\rangle\langle 0| \otimes\langle 0|), \\
A_{\top}\left[-\frac{1}{2}\right]=\frac{1}{\sqrt{3}}(Z \otimes\langle 0|-| 0\rangle\langle 1| \otimes\langle 1|), \\
B\left[+\frac{3}{2}\right]=|0\rangle\langle 1|, \\
B\left[-\frac{3}{2}\right]=-|1\rangle\langle 0|,
\end{gathered}
$$
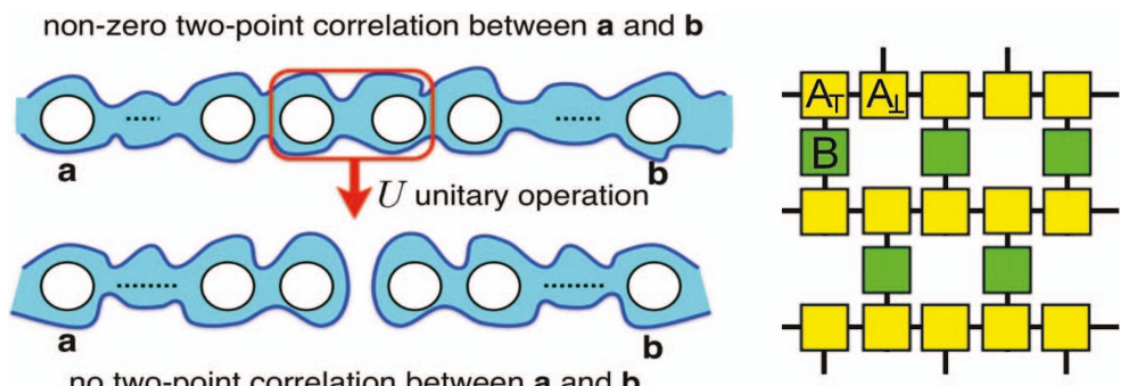

Figure 2 | Left: The AKLT state cannot be decomposed into two chains by a local unitary. Right: The tensor network of Ref. ${ }^{31}$. 


$$
\begin{gathered}
B\left[+\frac{1}{2}\right]=|1\rangle\langle 1|, \\
B\left[-\frac{1}{2}\right]=-|0\rangle\langle 0|
\end{gathered}
$$

(see Fig. 2 right). $A_{\perp}$ 's are defined in the same way. Each horizontal line works as a single-qubit wire. Two nearestneighbour horizontal chains are decoupled by measuring sites $\mathrm{B}$ in the $z$-basis. Before starting the computation, the filtering operation $\left\{F, F^{\prime}\right\}$, where

$F \equiv \frac{1}{\sqrt{3}}\left|\frac{3}{2}\right\rangle\left\langle\frac{3}{2}\left|+\frac{1}{\sqrt{3}}\right|-\frac{3}{2}\right\rangle\left\langle-\frac{3}{2}|+| \frac{1}{2}\right\rangle\left\langle\frac{1}{2}|+|-\frac{1}{2}\right\rangle\left\langle-\frac{1}{2}\right|$ and $F^{\prime} \equiv \sqrt{\frac{2}{3}}\left|\frac{3}{2}\right\rangle\left\langle\frac{3}{2}\left|+\sqrt{\frac{2}{3}}\right|-\frac{3}{2}\right\rangle\left\langle-\frac{3}{2}\right|$, is applied on each site A. Let us assume that the filtering is succeeded (i.e., $F$ is realized) and a site $B$ is projected onto $\left|\frac{3}{2}\right\rangle$. Then, the measurement

$$
\mathcal{M}_{\pi / 2, \phi} \equiv\left\{\frac{1}{\sqrt{2}}\left(\left|\frac{1}{2}\right\rangle \pm e^{i \phi}\left|-\frac{3}{2}\right\rangle\right),\left|-\frac{1}{2}\right\rangle,\left|\frac{3}{2}\right\rangle\right\}
$$

implements $Z X e^{i Z \phi / 2}, X e^{i Z \phi / 2}$, or $Z$, respectively. (The result $\left|\frac{3}{2}\right\rangle$ does not occur.) Let us assume that the singlequdit error

$$
\rho \rightarrow p E \rho E^{\dagger}+(1-p) \rho,
$$

where $E$ is the error which exchanges $\left|\frac{1}{2}\right\rangle$ and $\left|-\frac{1}{2}\right\rangle$, occurs, and that thus affected particle is measured in $\mathcal{M}_{\pi / 2, \phi}$. If the measurement result is $\left|-\frac{1}{2}\right\rangle$, we obtain the map

$$
\rho \rightarrow p|1\rangle\langle 0|\rho| 0\rangle\langle 1|+(1-p) Z \rho Z,
$$

which is not linear CPTP, is implemented in the correlation space.

\section{Methods}

Proof: In order to show Theorem, let us assume that

There is no such $\mathcal{E}$.

We will see that this assumption leads to the contradiction that $d \leq 2$. First, let us consider the state

$$
\left(I^{\otimes N-1} \otimes U_{1 \leftrightarrow 2}\right)|\Psi(L, R)\rangle_{1}^{N},
$$

where $U_{a \leftrightarrow b} \equiv|a\rangle\langle b|+| b\rangle\langle a|+I-| a\rangle\langle a|-| b\rangle\langle b|$ is the unitary error which exchanges $|a\rangle$ and $|b\rangle$, and $I$ is the identity operator on a single qudit. In Eq. (4), the error $U_{1 \leftrightarrow 2}$ is applied on the first physical qudit of $|\Psi(L, R)\rangle_{1}^{N}$. If we do the measurement $\mathcal{M}_{\theta, \phi}$ on the first physical qudit of Eq. (4), and if the measurement result is $|2\rangle$, Eq. (4) becomes

$$
\begin{array}{r}
\frac{1}{\sqrt{f_{N-1}(|L\rangle, A[1]|R\rangle)}}\|A[1]\| \sum_{k_{2}=0}^{d-1} \cdots \sum_{k_{N}=0}^{d-1}\langle L| A\left[k_{N}\right] \ldots \\
A\left[k_{2}\right] \frac{A[1]}{\|A[1]\|}|R\rangle\left|k_{N}, \ldots, k_{2}\right\rangle \otimes|2\rangle .
\end{array}
$$

In other words, the operation $|R\rangle \rightarrow \frac{A[1]}{\|A[1]\|}|R\rangle$ is implemented in the correlation space. By the assumption Eq. (3), this operation should work as a TP operation in the correlation space. Therefore,

$$
\frac{A^{\dagger}[1]}{\|A[1]\|} \frac{A[1]}{\|A[1]\|}=I
$$

By taking $\eta \equiv\|A[1]\|^{2}$,

$$
A^{\dagger}[1] A[1]=\eta I
$$

Second, let us consider the measurement $\mathcal{M}_{\theta, \phi}$ on the first physical qudit of $\left(I^{\otimes N-1} \otimes U_{0 \leftrightarrow 2} V^{s}\right)|\Psi(L, R)\rangle_{1}^{N}$, where $s \in\{0,1, \ldots, d-1\}, V \equiv \sum_{p=0}^{d-1} e^{-i \omega p}|p\rangle\langle p|$ is a unitary phase error, and $\omega \equiv 2 \pi / d$. If the measurement result is $\left|\alpha_{\theta, \phi}\right\rangle$,

$$
\left(e^{-2 i s \omega} \cos \frac{\theta}{2} A[2]+e^{-i(\phi+s \omega)} \sin \frac{\theta}{2} A[1]\right) / \sqrt{\gamma}
$$

is implemented in the correlation space, where

$\sqrt{\gamma} \equiv\left\|e^{-2 i s \omega} \cos \frac{\theta}{2} A[2]+e^{-i(\phi+s \omega)} \sin \frac{\theta}{2} A[1]\right\|$. By the assumption Eq. (3), this

should work as a TP operation in the correlation space. Therefore,

$$
\begin{aligned}
\gamma I= & \cos ^{2} \frac{\theta}{2} A^{\dagger}[2] A[2]+\sin ^{2} \frac{\theta}{2} A^{\dagger}[1] A[1] \sin \theta \\
& \left(e^{-i(\phi-s \omega)} A^{\dagger}[2] A[1]+e^{i(\phi-s \omega)} A^{\dagger}[1] A[2]\right) .
\end{aligned}
$$

By the assumption Eq. (2), $A^{\dagger}[2] A[2]=\xi I$, where $\xi \equiv\|A[2]\|^{2}$. Furthermore, as we have shown, $A^{\dagger}[1] A[1]=\eta I$ (Eq. (7)). Therefore,

$$
\gamma^{\prime} I=e^{-i(\phi-s \omega)} A^{\dagger}[2] A[1]+e^{i(\phi-s \omega)} A^{\dagger}[1] A[2],
$$

where $\gamma^{\prime} \equiv \frac{2}{\sin \theta}\left(\gamma-\xi \cos ^{2} \frac{\theta}{2}-\eta \sin ^{2} \frac{\theta}{2}\right)$. Finally, let us consider the measurement $\mathcal{M}_{\theta, \phi}$ on the first physical qudit of $\left(I^{\otimes N-1} \otimes U_{0 \leftrightarrow 1} U_{0 \leftrightarrow 2} V^{t}\right)|\Psi(L, R)\rangle_{1}^{N}$, where $t \in\{0$, $1, \ldots, d-1\}$. If the measurement result is $\left|\alpha_{\theta, \phi}\right\rangle$,

$$
\left(e^{-i t \omega} \cos \frac{\theta}{2} A[1]+e^{-i \phi-2 i t \omega} \sin \frac{\theta}{2} A[2]\right) / \sqrt{\delta}
$$

is implemented in the correlation space, where $\sqrt{\delta} \equiv\left\|e^{-i t \omega} \cos \frac{\theta}{2} A[1]+e^{-i \phi-2 i t \omega} \sin \frac{\theta}{2} A[2]\right\|$. By the assumption Eq. (3), this should also work as a TP operation in the correlation space. Therefore,

$$
\delta^{\prime} I=e^{i(\phi+t \omega)} A^{\dagger}[2] A[1]+e^{-i(\phi+t \omega)} A^{\dagger}[1] A[2],
$$

where $\delta^{\prime} \equiv \frac{2}{\sin \theta}\left(\delta-\xi \sin ^{2} \frac{\theta}{2}-\eta \cos ^{2} \frac{\theta}{2}\right)$. From Eqs. (8) and (9), $\epsilon I=\left[e^{-2 i(\phi-s \omega)}-\right.$ $\left.e^{2 i(\phi+t \omega)}\right] A^{\dagger}[2] A[1]$, where $\epsilon \equiv e^{-i(\phi-s \omega)} \gamma^{\prime}-e^{i(\phi+t \omega)} \delta^{\prime}$. Let us assume that $e^{-2 i(\phi-s \omega)}-e^{2 i(\phi+t \omega)}$ $\neq 0$. Then, $\epsilon^{\prime} I=A^{\dagger}[2] A[1]$, where $\epsilon^{\prime} \equiv \frac{\epsilon}{e^{-2 i(\phi-s \omega)}-e^{2 i(\phi+t \omega)}}$. If $\epsilon^{\prime}=0, A^{\dagger}[2] A[1]=$ 0 , which means $A[1]=0$ since $A[2]$ is unitary up to a constant (assumption Eq. (2)). Therefore, $\epsilon^{\prime} \neq 0$. In this case, $A[1]=\epsilon^{\prime \prime} A[2]$ for certain $\epsilon^{\prime \prime} \neq 0$, since $A[2]$ is unitary up to a constant. Hence $e^{-2 i(\phi-s \omega)}-e^{2 i(\phi+t \omega)}=0$. This means

$$
2 \phi+(t-s) \omega=r_{s, t} \pi,
$$

where $r_{s, t} \in\{0,1,2,3, \ldots\}$. Let us take $t=s=0$. Then, Eq. (10) gives $\phi=r_{0,0} \frac{\pi}{2}\left(r_{0,0} \in\right.$ $\{0,1,2, \ldots\})$. Let us take $s=1, t=0$. Then, Eq. (10) gives $\phi=\frac{\pi}{d}+r_{1,0} \frac{\pi}{2}\left(r_{1,0} \in\{0,1,2\right.$, $\ldots\})$. In order to satisfy these two equations at the same time, there must exist $r_{0,0}$ and $r_{1,0}$ such that $r_{0,0} \frac{\pi}{2}=\frac{\pi}{d}+r_{1,0} \frac{\pi}{2}$. If $r_{0,0}=r_{1,0}$, then $0=1 / d$ which means $d=\infty$. Therefore $r_{0,0} \neq r_{1,0}$. Then we have $d=\frac{2}{r_{0,0}-r_{1,0}} \leq 2$, which is the contradiction. One might think that if we rewrite the post-measurement state Eq. (5) as

$$
\begin{aligned}
& \frac{1}{\sqrt{f_{N-1}(|L\rangle, A[1]|R\rangle)}} \| A[1]|R\rangle \| \\
& \sum_{k_{2}=0}^{d-1} \ldots \sum_{k_{N}=0}^{d-1}\left\langle L\left|A\left[k_{N}\right] \ldots A\left[k_{2}\right] \frac{A[1]}{\| A[1]|R\rangle \|}\right| R\right\rangle\left|k_{N}, \ldots, k_{2}\right\rangle \otimes|2\rangle
\end{aligned}
$$

and redefine the operation implemented in the correlation space as $|R\rangle \rightarrow \frac{A[1]}{\| A[1]|R\rangle \|}|R\rangle$, the TP-ness is recovered in the correlation space. However, in this case, the non-lineally appears unless $A^{\dagger}[1] A[1] \propto I$, and therefore if we require the linearity in the correlation space, we obtain the same contradiction.

In short, if $d \geq 3$ not all physical errors on physical qudits appear as linear CPTP errors in the correlation space of pure matrix product states.

1. Raussendorf, R. \& Briegel, H. J. A one-way quantum computer. Phys. Rev. Lett. 86, 5188 (2001).

2. Raussendorf, R., Browne, D. E. \& Briegel, H. J. Measurement-based quantum computation on cluster states. Phys. Rev. A 68, 022312 (2003).

3. Raussendorf, R. Ph.D. thesis, Ludwig-Maximillians Universität München, 2003.

4. Gross, D. \& Eisert, J. Novel schemes for measurement-based quantum computation. Phys. Rev. Lett. 98, 220503 (2007).

5. Gross, D., Eisert, J., Schuch, N. \& Perez-Garcia, D. Measurement-based quantum computation beyond the one-way model. Phys. Rev. A 76, 052315 (2007).

6. Gross, D. \& Eisert, J. Quantum computational webs. Phys. Rev. A 82, 040303(R) (2010). 
7. Fannes, M, Nachtergaele, B. \& Werner, R. F. Valence bond states on quantum spin chains as ground states with spectral gap. J. Phys. A 24, L185 (1991).

8. Verstraete, F., Cirac, J. I. \& Murg, V. Matrix product states, projected entangled pair states, and variational renormalization group methods for quantum spin systems. Adv. Phys. 57, 143 (2008).

9. Cirac, J. I. \& Verstraete, F. Renormalization and tensor product states in spin chains and lattices. J. Phys. A: Math. Theor. 42, 504004 (2009).

10. Cai, J. M., Dür, W., Van den Nest, M., Miyake, A. \& Briegel, H. J. Quantum computation in correlation space and extremal entanglement. Phys. Rev. Lett. 103, 050503 (2009).

11. Morimae, T. How to upload a physical quantum state into correlation space. Phys. Rev. A 83, 042337 (2011).

12. Fujii, K. \& Morimae, T. Computational power and correlation in a quantum computational tensor network. Phys. Rev. A 85, 032338 (2012).

13. Shor, P. W. Proc. of the 37th Symposium on Foundations of Computing, p.56 (IEEE Computer Society Press, 1996).

14. Aharonov, D. \& Ben-Or, M. Proc. of the 29th Annual ACM Symposium on Theory of Computing, p. 176 (ACM Press, New York, 1998).

15. Kitaev, A. Yu. Russian Math. Surveys 52, 1191 (1997)

16. Knill, E., Laflamme, R. \& Zurek, W. H. Resilient quantum computation: error models and thresholds. Proc. Roy. Soc. London, Ser A 454, 365 (1998).

17. Terhal, B. M. \& Burkard, G. Fault-tolerant quantum computation for local nonMarkovian noise. Phys. Rev. A 71, 012336 (2005).

18. Nielsen, M. A. \& Dawson, C. M. Fault-tolerant quantum computation with cluster states. Phys. Rev. A 71, 042323 (2005).

19. Aliferis, P. \& Leung, D. W. Simple proof of fault tolerance in the graph-state model. Phys. Rev. A 73, 032308 (2006).

20. Silva, M., Danos, V., Kashefi, E. \& Ollivier, H. A direct approach to fault-tolerance in measurement-based quantum computation via teleportation. New. J. Phys. 9, 192 (2007)

21. Fujii, K. \& Yamamoto, K. Topological one-way quantum computation on verified logical cluster states. Phys. Rev. A 82, 060301(R) (2010).

22. Fujii, K. \& Yamamoto, K. Cluster-based architecture for fault-tolerant quantum computation. Phys. Rev. A 81, 042324 (2010).

23. Raussendorf, R. \& Harrington, J. Fault-tolerant quantum computation with high threshold in two dimensions. Phys. Rev. Lett. 98, 190504 (2007).

24. Raussendorf, R., Harrington, J. \& Goyal, K. Topological fault-tolerance in cluster state quantum computation. New J. Phys. 9, 199 (2007).

25. Li, Y., Browne, D. E., Kwek, L. C., Raussendorf, R. \& Wei, T. C. Thermal states as universal resources for quantum computation with always-on interactions. Phys. Rev. Lett. 107, 060501 (2011).
26. Fujii, K. \& Morimae, T. Topologically protected measurement-based quantum computation on the thermal state of a nearest-neighbor two-body Hamiltonian with spin-3/2 particles. Phys. Rev. A 85, 010304(R) (2012).

27. Chen, J., Chen, X., Duan, R., Ji, Z. \& Zeng, B. No-go theorem for one-way quantum computing on naturally occurring two-level systems. Phys. Rev. A 83, 050301(R) (2011).

28. Brennen, G. K. \& Miyake, A. Measurement-based quantum computer in the gapped ground state of a two-body Hamiltonian. Phys. Rev. Lett. 101, 010502 (2008).

29. Affleck, I., Kennedy, T., Lieb, E. H. \& Tasaki, H. Valence bond group states in isotropic quantum antiferromagnets. Comm. Math. Phys. 115, 477 (1988).

30. Chen, X., Zeng, B., Gu, Z., Yoshida, B. \& Chuang, I. L. Gapped two-body Hamiltonian whose unique ground state is universal for one-way quantum computation. Phys. Rev. Lett. 102, 220501 (2009).

31. Cai, J. M., Miyake, A., Dür, W. \& Briegel, H. J. Universal quantum computer from a quantum magnet. Phys. Rev. A 82, 052309 (2010).

\section{Acknowledgements}

TM acknowledges supports by ANR (StatQuant, JC07 07205763) and JSPS. KF acknowledges support by MEXT (Grant-in-Aid for Scientific Research on Innovative Areas 20104003).

\section{Author contributions}

All results are obtained through the discussion between TM and KF. Mainly TM wrote the manuscript. All authors reviewed the manuscript.

\section{Additional information}

Supplementary information accompanies this paper at http://www.nature.com/ scientificreports

Competing financial interests: The authors declare no competing financial interests.

License: This work is licensed under a Creative Commons

Attribution-NonCommercial-ShareAlike 3.0 Unported License. To view a copy of this license, visit http://creativecommons.org/licenses/by-nc-sa/3.0/

How to cite this article: Morimae, T. \& Fujii, K. Not all physical errors can be linear CPTP maps in a correlation space. Sci. Rep. 2, 508; DOI:10.1038/srep00508 (2012). 